\title{
Research on the Microbial Community Structure of Wooden Relics and the Preservation of Water Environment: Illumina MiSeq Sequencing
}

\author{
Liu Tang1, Jingjing Xing1, Qiong Lei², Zuming Qiu², Jun Zhang³, Li'an Ma' ${ }^{*}$ \\ ${ }^{1}$ College of Life Science, Yangtze University, Jingzhou, China \\ ${ }^{2}$ Jingzhou Conservation Center, Jingzhou, China \\ ${ }^{3}$ Guangdong Institute of Microbiology, Guangzhou, China \\ Email: *malian@yangtzeu.edu.cn
}

How to cite this paper: Tang, L., Xing, J.J., Lei, Q., Qiu, Z.M., Zhang J. and Ma, L.A. (2019) Research on the Microbial Community Structure of Wooden Relics and the Preservation of Water Environment: Illumina MiSeq Sequencing. Open Access Library Journal, 6: e5315.

https://doi.org/10.4236/oalib.1105315

Received: March 6, 2019

Accepted: March 22, 2019

Published: March 25, 2019

Copyright $\odot 2019$ by author(s) and Open Access Library Inc.

This work is licensed under the Creative Commons Attribution International License (CC BY 4.0).

http://creativecommons.org/licenses/by/4.0/

\begin{abstract}
[Objective] Microorganisms are an important disease in archaeological wood lacquer. The effect of single microorganism on the erosion of wooden relics is small, but the microbial community is more harmful to the wooden relics. Therefore, it is very important to acquaint the microbial community structure of wooden relics and their preservation environment. [Methods] Illumina MiSeq sequencing method was used in this study to explore wood relics and full water environmental microbial community structure. [Results] The results showed that the level of wood samples was distributed in 17 phyla containing 6 dominant bacteria, and water samples in 21 containing 7 dominant phyla. In the family level, the wood samples were distributed in 64 with 8 dominant families, while the water samples were distributed in 93 with 10 dominant families. At the level of the genus, the wood samples were distributed in 72 with 8 dominant genera, while the water samples were distributed in 105 with 10 dominant genera. [Conclusion] Most of the microbes in the wood samples are the same as those in the water environment, but there are differences in the community distribution.
\end{abstract}

\section{Subject Areas \\ Environment Science}

\section{Keywords}

Illumina MiSeq Sequencing, Wood Lacquer, Microbial Community, Dominant Phylum, Dominant Family, Dominant Genera 


\section{Introduction}

A lot of research shows that bacteria can use carbohydrates from wood to survive in a long-term anoxic subterranean environment [1] [2] [3]. Most of the wooden relics in the middle and south of China are in the full water environment [4], so the method of immersion preservation is adopted after unearthed. Wooden relics are thought corroded by microorganisms after they are unearthed [5] [6], so it is necessary to know the diversity of microbial communities in wood lacquers.

At present, researchers judge the microbes in wooden relics mainly by separation, purification and identification, which deviate greatly from the original habitat (accounting for only $0.1 \%$ to $10 \%$ of the total number of environmental microbes) [7]. In order to solve this problem, DNA fingerprinting technology, phospholipid fatty acid analysis, gene chip and other microbial community detection methods came into being, but all have the shortcomings of low flux and small information volume [8]. High throughput sequencing has many advantages, such as wide coverage, high accuracy, low cost, long sequencing and bidirectional sequencing [9].

Using Illumina MiSeq sequencing characteristics to understand the microbial community structure of wood lacquer preserved with water is of great significance for analyzing the corrosion causes and disease information of wood lacquer.

\section{Materials and Methods}

\subsection{Sampling and Sample Handling}

Samples were collected with sterilizing tweezers from the waterlogged wooden lacquer (No.F446) in Jingzhou Museum 0.3 - $0.5 \mathrm{~g}$ sample was cut, ground, and added with sterile water at 1:10 ratio. Suspensions were extracted after centrifugation for several times. Then the processed samples were stored at $4^{\circ} \mathrm{C}$. The water samples were named $\mathrm{W} 1, \mathrm{~W} 2, \mathrm{~W} 3, \mathrm{~W} 4$, and the wood samples were named W5, W6, W7, W8.

\subsection{DNA Extraction, Polymerase Chain Reaction and Illumina MiSeq Sequencing}

DNA was extracted from samples using the improved method for CTAB [10]. The extracted DNA was amplified using a set of bar-coded primers $515 \mathrm{~F}$ and 806R. The PCR products from each sample were pooled together with equal molar concentrations, and then sequenced within Illumina Miseq platform.

\subsection{Sequencing Analyses}

The sample DNA was sent to the biological Mdt InfoTech Ltd. of NOA, Beijing, to carry out high throughput sequencing of Illumina MiSeq 2500. The sequencing results were compared with the gene bank of NCBI, and the bacteria were divided into different OTUs based on the homology of $97 \%$. In order to evaluate 
the diversity of microbial community composition, cluster analysis was conducted on the detected OTU based on the Bray-Curtis distance. Using QIIME software to calculate the Alpha diversity index of the sample, including the Chaol value and the Shannon index [11].

\section{Results and Analyse}

\subsection{Quality Analysis of Sequencing Results}

A total of 178,312 raw sequences were obtained; after filtering, 177,847 high-quality 16SrRNA gene sequences with an average length of 251 - 254 bp were recovered.

\subsection{Analysis of Alpha Diversity in Microbial Communities}

The diversity of microbial community is related to the number of microorganisms and the uniformity of distribution. Figure 1 shows that the Shannon index and pielou evenness of water samples are larger than those of wood samples. However, the Simpson index of wood samples is larger than that of water samples. Therefore, the species of microorganisms in water samples were larger than those in wood samples, but the distribution of microorganisms in wood samples is even.

\subsection{Sample Community Composition Analysis}

\subsubsection{Analysis of Microbial Community Composition at Phylum Level} Wood samples were distributed on 17 phyla, and water samples were distributed on 21 phyla. In addition, wood samples had 6 dominant phyla (relative abundance $>1 \%$ ), while 7 dominant phyla (relative abundance $>1 \%$ ) were found in

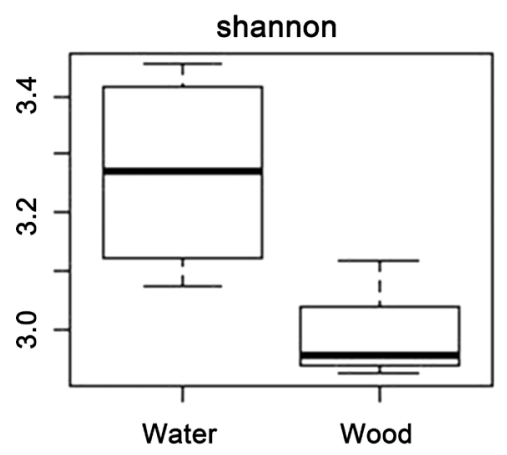

pielou.evenness

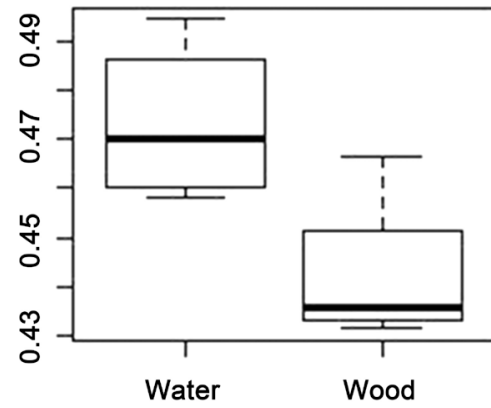

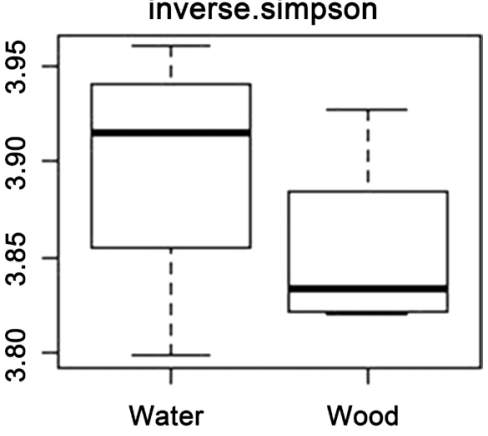

simpson.evenness

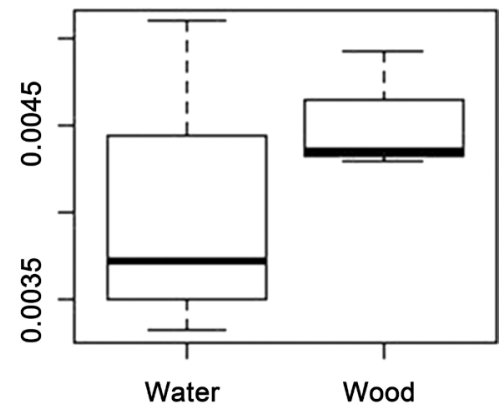

Figure 1. Alpha-diversity of samples. 
water samples. As can be seen from Figure 2, there is a certain difference in the distribution of microbes in the wood samples and water samples in phylum. The relative abundance of Acidobacteria in wood samples was extremely significantly than that in water samples $(P<0.05)$, and the relative abundance of Planctomycetes and Nitrospira in water samples was extremely significantly than that in wood samples $(P<0.01)$.

\subsubsection{Analysis of Microbial Community Composition at Family Level}

The bacteria in the water samples are distributed in 93 families, and the wood samples are distributed in 64 families. In addition, wood samples had 8 dominant families (relative abundance $>1 \%$ ), while 10 dominant families (relative abundance $>1 \%$ ) were found in water samples. Water samples and wood samples in 11 families with significant differences among the relative abundance > $1 \%$ for a total of six families. Respectively: Caulobacteraceae, Hyphomicrobiaceae, Planctomycetaceae, Sinobacteraceae, Nitrospiraceae, Erythrobacteraceae. Figure 3 shows that there are differences in the community structure distribution of the eight samples. But the internal differences of wood samples and water samples are small.

\subsubsection{Analysis of Microbial Community Composition at Genus lEvel}

The total number of bacteria at genus in wood samples is 72 , and in water samples is 104 . The dominant genera in (relative abundance $>1 \%$ ) the wood sample are 8 , and the proportion is $42.15 \%$. The dominant genera in water sample is 10 , and the proportion is $30.60 \%$. The rare genera (relative abundance $<0.1 \%$ ) in the wood sample are 48 and the proportion is $1.45 \%$. And the water sample is 66 and the proportion is $1.90 \%$. It is known from Table 1 that there is no significant difference between the wood samples and the water samples in the distribution of the dominant genus. However, there are significant differences in the distribution of all the bacteria at genus and the rare bacteria in the wood and water samples. In addition, there is a significant difference in the proportion of the distribution of the dominant and the rare genus. It suggests that the distribution of wood samples and water samples is great different in the distribution of bacteria.

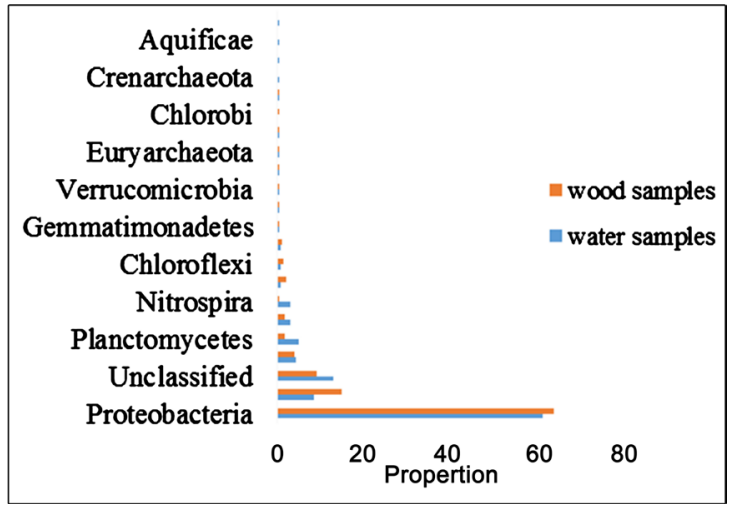

Figure 2. Comparison of wood samples and water environment on the distribution in phylum. 


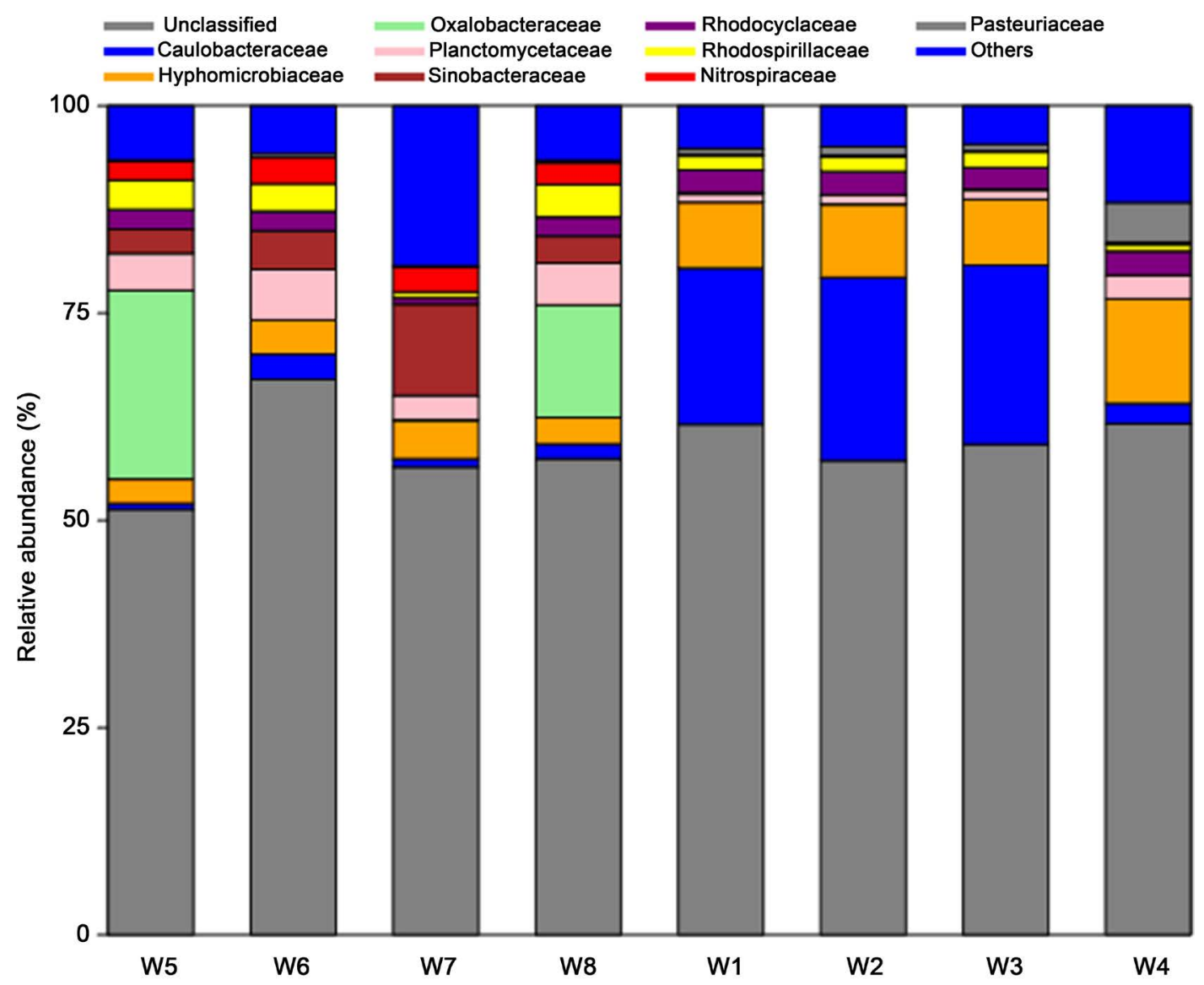

Figure 3. Analysis of the composition of community structure of wood samples and water samples.

Table 1. The distribution of the bacteria in wood samples and water samples.

\begin{tabular}{cccccc}
\hline & Total number & $\begin{array}{c}\text { Number of } \\
\text { dominant genus }\end{array}$ & $\begin{array}{c}\text { Proportion of } \\
\text { dominant genus (\%) }\end{array}$ & $\begin{array}{c}\text { Number of } \\
\text { rare genus }\end{array}$ & $\begin{array}{c}\text { Proportion of } \\
\text { rare genus (\%) }\end{array}$ \\
\hline W1 & 73 & 7 & 42.29 & 49 & 1.44 \\
W2 & 76 & 6 & 43.95 & 52 & 1.34 \\
W3 & 70 & 6 & 43.6 & 49 & 1.59 \\
W4 & 69 & 12 & 38.76 & 43 & 1.42 \\
W5 & 92 & 9 & 37.39 & 51 & 1.9 \\
W6 & 104 & 9 & 22.64 & 63 & 1.92 \\
W7 & 98 & 11 & 30.72 & 68 & 1.67 \\
W8 & 124 & 11 & 31.65 & 80 & 2.12 \\
$\begin{array}{c}\text { P-Value } \\
\text { (P }<0.05)\end{array}$ & 0.004 & 0.196 & 0.012 & 0.034 & 0.005 \\
\hline
\end{tabular}

\subsection{Analysis of Beta Diversity in Microbial Communities}

DCA analysis method was adopted to this research, because it can not only analyze the data matrix composed of coverage and quadrat, but also can analyze data matrix by frequency and quadrat, biomass and composition analysis of the 
sample [12]. As shown in Figure 4, the wood samples and water samples are clearly divided into two categories. In addition, the four sampling points of the wood sample are more concentrated than the water samples. It suggests that there is a certain difference between the microbial community structure in the wood samples and the water samples.

\section{Discussion}

The unearthed lacquer is kept in a full water environment and will be affected by the microorganism in the water environment. Based on Illumina MiSeq sequencing, the bacteria in the wood samples were detected in 17 bacteria phyla and 94 families and 106 bacteria genera while the bacteria in the wood samples were detected in 21 bacteria phyla and 65 families and 72 bacteria genera. Through calculation and analysis, there are 6 dominant phyla and 10 dominant families and 10 dominant genera in water samples, and the wood samples contain 7 dominant phyla, 8 dominant families and 8 dominant genera. The results showed that the smaller the classification level, the diversity of microbial community structure diversity between water samples and wood samples are greater. And there are significant differences in the microbial community structure of the two samples at the level of the genus.

Many scholars have done the study of microorganism on wooden lacquer. ZJ Zhao [13] and others conducted microbial studies on the site of the Chengdu coffin, and isolated Pseudomonas, Flavobacterium, Proteur, and Cellulomonas. [14] detected the bacteria in the burial environment of the Han Tombs in Siyang,

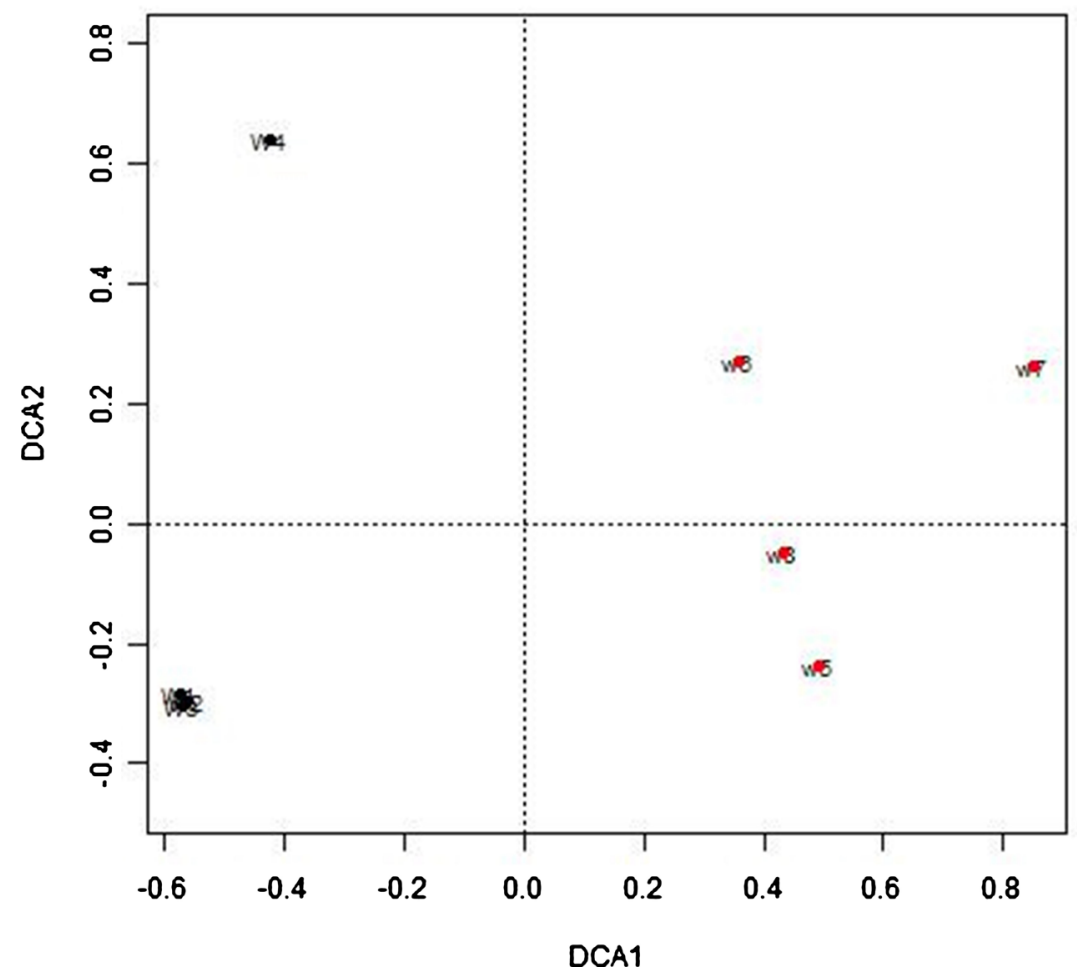

Figure 4. DCA analysis of wood samples and water samples. 
and found the presence of Pseudomonas. Furthermore, [15] studied bacterial diversity of waterlogged in 108 samples by DGGE; the results showed that $C y$ tophaga-Flavobacterium-Bacteroides (CFB) complex and the Pseudomonas group were commonly recovered, with relatives of the Cellvibrio and Brevundimonas groups also present, and most of these bacteria were detected in this study. The distribution of microbes in the lacquer of the full water preservation wood has regional differences and similarity.

Moreover, [16] isolated and identified the bacteria on the wooden lacquer F446 and the bacteria in the water conservation environment. The bacteria isolated from the wood samples belonged to 5 genera, the dominant bacteria were Bacillus, the bacteria isolated from the water samples belonged to 9 genera, and the dominant bacteria were Brevis. [17] selected 32 strains of bacteria from F455 wood lacquer belonging to 4 genera, the dominant genus was Bacillus, and 9 species of bacteria were in water samples, of which 9 were Brevis.

At present, the study of microbial community of full water preserved wooden lacquer in world is still in a relatively blank stage. However, by microorganism pure culture method, after isolation and identification, it can be found that the microbial diversity of water lacquer wares is much larger than that of wood samples. Therefore, in order to delay the corrosion process of wooden lacquer, it is suggested to preserve the wooden lacquer with aseptic water, and regularly replace the water to keep the number of microbes in the water environment in a low state.

\section{Conflicts of Interest}

The authors declare no conflicts of interest regarding the publication of this paper.

\section{References}

[1] Macchioni, N., Capretti, C., Sozzi, L. and Pizzo, B. (2013) Grading the Decay of Waterlogged Archaeological Wood According Toanatomical Characterisation. The Case of the Fiavé Site (N-E Italy). International Biodeterioration \& Biodegradation, 84, 54-64. https://doi.org/10.1016/j.ibiod.2013.05.028

[2] Pedersen, N.B., Björdal, C.G., Jensen, P. and Felby, C. (2013) Order XIII. Bacterial Degradation of Archaeological Wood in Anoxic Waterlogged Environments. In: Order Stability of Complex Carbohydrate Structure: Biofuels, Foods Vaccines and Shipwrecks, Spec. Pub. Royal Soc. of Chem, London, 160-187.

[3] Clausen, C.A. (1996) Bacterial Associations with Decaying Wood: A Review. International Biodeterioration \& Biodegradation, 37, 101-107. https://doi.org/10.1016/0964-8305(95)00109-3

[4] Chen, J.C., Huang, X., Chen, X.L. and Chen, Z.X. (2015) Corrosion Type and Conservation of Archaeological Waterlogged Wood. Materials Review, 29, 96-101.

[5] Xu, R.L. (2013) Advances in Bacterial Diseases and Diagnostic Techniques of Waterlogged Woody Artifacts. China Cultural Heritage Scientific Research, 25, 104-110.

[6] Liu, L. (2014) Microbiological Corrosion and Anti-Corrosion Measures for Waterlogged Wood Objects. Chinese Cultural Relics, 4, 83-85. 
[7] Che, Y.L., Wang, H., Hu, H.Y., Liang, W. and Guo, Y.F. (2005) Research Progress on Analytical Technologies Used in Microbial Community Structure and Diversity. Ecology \& Environmental Sciences, 14, 127-133.

[8] Lou, J., Liu, Y. and Li, Y. (2014) Review of High-Throughput Sequencing Techniques in Studies of Soil Microbial Diversity. Chinese Agricultural Science Bulletin, 30, 256-260.

[9] Qin, N., Li, D.F. and Yang, R.F. (2011) High Throughput Sequencing Technology and Its Application in Microbiology Research. Acta Microbiologica Sinica, 51, 445-457.

[10] Xing, J.J., Lei, Q., Qiu, Z.M., Zhang, J. and Ma, L.A. (2018) Research on Microbial Community Structure and Diversity in the Waterlogged Archaeological Wood and Lacquer Named F446 by Illimina MiSeq Technology.

[11] Oren, A., Jiao, Z.W., Janbolat, Mu, G.L. and Yang, X.R. (2017) Determination of Bacteria Diversity of Degraded Grassland in Xinyuan County by High-Throughput Sequencing Technology. Microbiology China, 44, 545-553.

[12] Jia, X.N., Cheng, J.M. and Wan, H.E. (2007) Application Present Situation of DCA, CCA, and DCCA Ordination of Grassland Vegetation Communities in China. Chinese Agricultural Science Bulletin, 23, 391-394.

[13] Zhao, Z.J., Xiao, L. and Sun, J. (2005) 4th Annu. Conf. of Chinese Cultural. Relics Pro. Tech. Asso., 381-393.

[14] Zhang, J.P., Xi, S.C. and Zhou, J.L. (2005) Analysis and Detection of Bacteria in Burial Environment of Han Tombs in Siyang. Sciences of Conservation and Archaeology, 17, 36-40.

[15] Landy, E.T., Mitchell, J.I., Hotchkiss, S. and Eaton, R.A. (2008) Bacterial Diversity Associated with Archaeological Waterlogged Wood: Ribosomal RNA Clone Libraries and Denaturing Gradient Gel Electrophoresis (DGGE). International Biodeterioration \& Biodegradation, 61, 106-116. https://doi.org/10.1016/j.ibiod.2007.07.007

[16] Lei, Q., Zhang, J., Qiu, Z.M., Jiang, T. and Ma, L.A. (2018) Identification of Cultured Bacteria Separated from an Excavated Waterlogged Archaeological Wood. Microbiology China, 45, 1-9.

[17] Zhang, J., Lei, Q., Qiu, Z.M., Fan, X.D., Wang, J., Zhan, Y.B. and Ma, L.A. (2017) Study of Wood Degradation by Bacteria from the Archaeological Waterlogged wood f455 Excavated at Chu Tomb No.1 at Tianxingguan. Sciences of Conservation and Archaeology, 29, 19-36. 